\title{
IMPLEMENTATION OF A REHABILITATION ROBOT FOR GLENOHUMERAL JOINT MOBILIZATION
}

\author{
Jia-Ren Chang Chien \\ Department of Electronic Engineering \\ National Kaohsiung First University of Science and \\ Technology , Taiwan, ROC \\ ccjr0215@gmail.com
}

\begin{abstract}
In this study, we designed a robot with six degrees of freedom (six-DOF robot) to treat patients with glenohumeral joint (GHJ) disease by using data obtained from a strength sensor and the degree of displacement of the humerus. From a safety perspective, Grade III joint mobilization is conducted to realize a system design capable of being applied for observation and rehabilitation of patients with GHJ diseases.

The robot uses the output strength to displace the joint and reduce the resistance from joint tissue of the frozen shoulder. In the experiment, after dorsal translation mobilization (DTM) for all the subjects, from the load-displacement curve of GHJ, we could see progress of $0.59-3.31 \mathrm{~mm}$ for all subjects (under $30 \mathrm{~N}$ ). After five DTM procedures, the maximum displacement reached a stable condition.
\end{abstract}

Keywords: robot, dorsal translation mobilization, glenohumeral joint

\section{INTRODUCTION}

Frozen shoulder, also called adhesive capsulitis, was first introduced by Codeman (1934). It usually stems from pathological changes of the GHJ that limit the motions of the joint and can cause the patient intolerable pain at the affected areas. Frozen shoulder is divided into primary and secondary frozen shoulders. Currently, the cause of primary frozen shoulder remains unknown, whereas there are several possible causes of secondary frozen shoulder:[1] (a) Patients with diabetes. The incidence rate of frozen shoulder among healthy people is $2 \%-5 \%$ whereas the incidence rate in patients with diabetes is $10 \%-20 \%$. Up to $36 \%$ of these patients have insulin-dependent diabetes.(b) Those with injuries to the arms or upper limbs are prone to suffer frozen shoulder.(c) Those with a sedentary lifestyle. Some injuries resulting from the lack of exercise may also increase the probability of frozen shoulder. Clinically, frozen shoulder can be divided into four stages: pre-adhesive stage, freezing stage, frozen stage, and thawing stage. The pre-adhesive stage occurs in the first three months, and the patient will feel pain mainly when the joint activity reaches the terminal. The freezing stage occurs within 3-9 months after onset. The patients will suffer sustained and significant pain, and

\author{
Chin-Fu Chang, Jia-hau Shiu \\ Department of Electronic Engineering \\ National Kaohsiung First University of Science and \\ Technology, Taiwan, ROC \\ thickwater0@gmail.com \\ jhs.8901.3737@gmail.com
}

joint activities will be severely limited. The frozen stage occurs 9-15 months after the onset, and all joint activities will be severely limited, but the pains in the arms will be reduced. The thawing stage occurs within 15-24 months after the onset. During this stage, the patient will feel little if any pain. The joint activities will gradually improve, but if there is no proper treatment, the degree of joint activity will not return to normal.[2-3]

\section{BACKGROUND AND FEATURES}

Presently, there are many physical treatments for frozen shoulder, including oral medication, steroid injection, exercise therapy, and joint mobilization. Joint mobilization has been shown to be effective in relieving pain, increasing joint mobility, adjusting the joint structure, and alleviating muscle contracture. However, during the treatment of joint mobility, doctors should be aware of the clinical characteristics of frozen shoulder to set the treatment plan. In different stages, the targets, contents, and required dose of treatment are different. Clinically, the degrees of patients' pain, joint activity, and disability are divided into:[4]

a) High Irritability: the patient's pain is the most serious, and on the visual analog scale, the degree of pain is greater than $7 / 10$. The pain will affect a patient's rest or sleep, and the joint activity will be at the lowest degree. Under such circumstances, a doctor can only perform Grade I and II joint mobilizations for the patient.

b) Moderate Irritability: the degree of patient's pain is moderate. On the visual analog scale, the degree of pain is between $4 / 10$ and $6 / 10$. The patient will feel pain when the joint activity reaches the terminal position. A doctor may perform Grade II and III joint mobilizations.

c) Low Irritability: most of the patient's joints are stiff. On a visual analog scale, the degree of pain is lower than 3/10. A doctor may need to consider performing Grade II and Grade IV joint mobilizations.

In this article, we introduce a force control method (FCM) in which the robotic arm follows the control of the therapist, so the robot can implement GHJ mobilization. 


\section{THE FCM ALGORITHM}

The FCM algorithm uses a three-direction force sensor to control the displacement of the robotic arm with a stepping method. The concept of the FCM is shown in Fig. 1. First, the forces in the three directions obtained using the sensor are transformed into a unit vector as the offset of the movement of the robotic arms. Secondly, the input resultant force and reference value are compared. If the resultant force is smaller than the reference value, the work is stopped. If the resultant force is greater than the reference value, then movement proceeds according to the calculated value.[5]

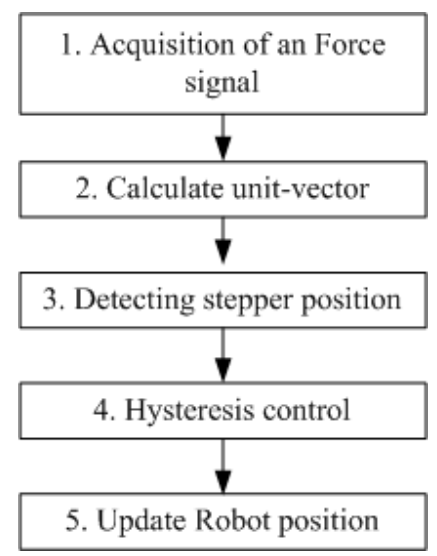

FIG. 1. The FCM Algorithm.

\section{SYSTEM IMPLEMENTATION}

The capsuloligamentous complex of GHJ has a fixing effect on all directions of the joint. Thus, when the capsule is adhesive, it will immobilize GHJ. We need only improve the ligament ductility of a part of the directions to improve the degree of activity of the entire joints. The structure of GHJ is displayed in Fig. 2. The experiment is mainly trying to let the robotic arm conduct dorsal translation mobilization (DTM) for the subjects' GHJ. During the experiment, the arms of the subjects are placed into a neutral position, and the therapist brings the robotic arm above the greater tubercle to allow the robotic arm to conduct DTM.

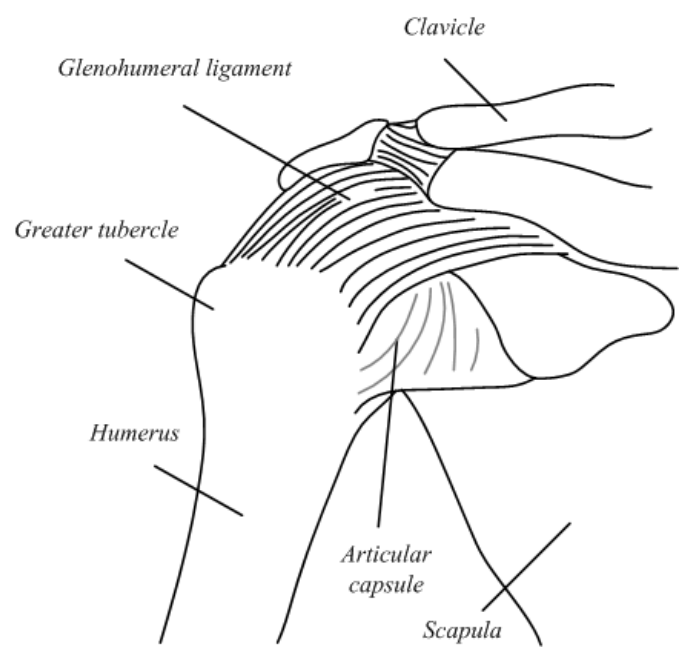

FIG. 2. Structure of GHJ.

A block diagram of the rehabilitation robot system is shown in Fig. 3. The entire hardware includes the six-DOF robot, control server, six-axis sensor, force measurement circuit, and PC. The six-DOF robot TX60 (shown as $\mathbb{A}$ in Fig. 5), produced by Stäubli Company, has a spherical stretching range of $670 \mathrm{~mm}$, a high repeat accuracy of \pm 0.02 $\mathrm{mm}$ and a maximum load capacity of $9 \mathrm{~kg}$. The TX60

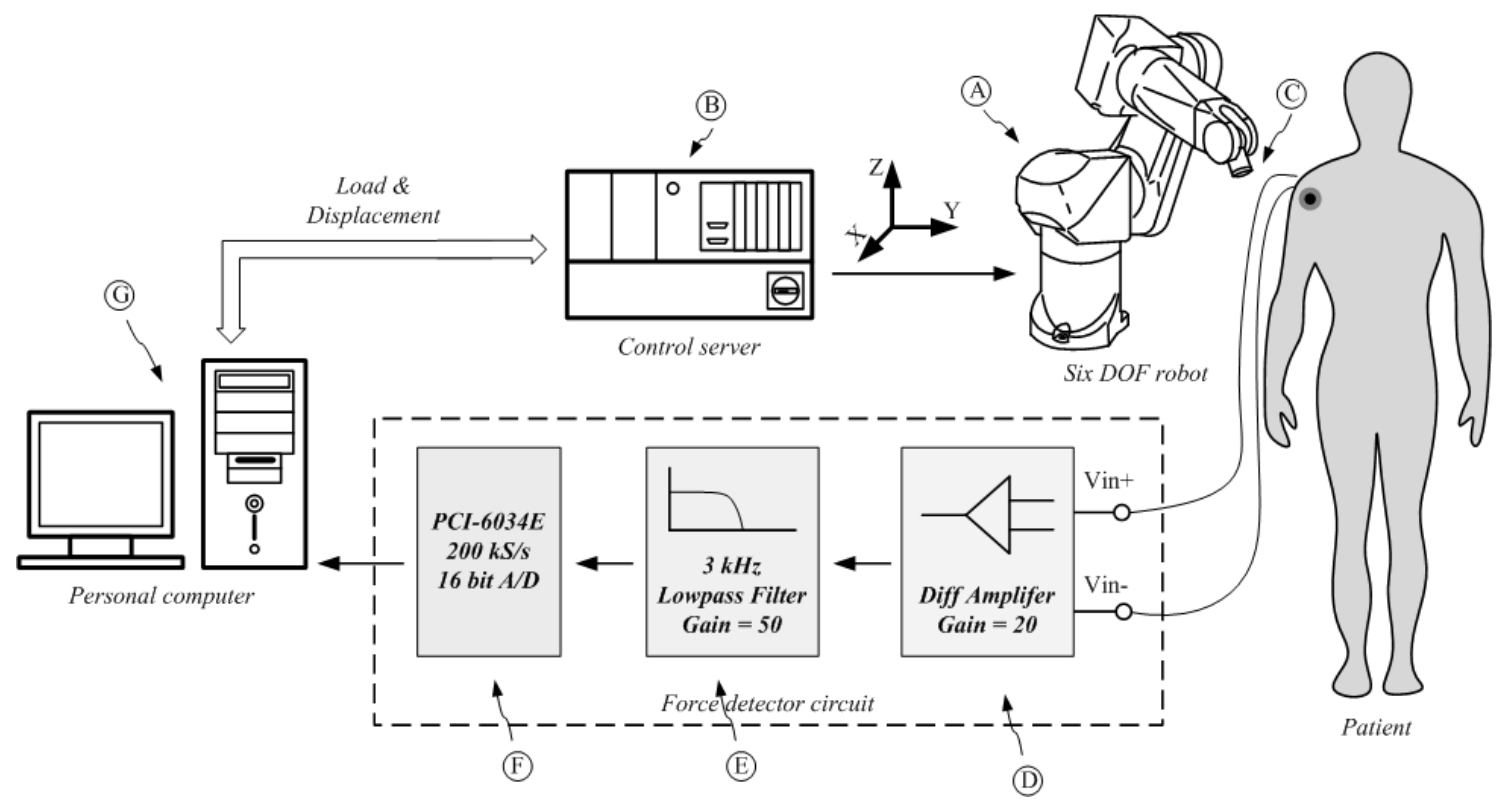

FIG. 3. The System Block Diagram. 
control system is run on a CS8C control server (B) with a built-in, real-time, multi-tasking operating system using the VAL3 language.

Firstly, the resistance force generated by the displacement of the humerus is measured by AMTI's MC3A-100 six-axis transducer. The load cell is installed on the end of the sixth axis of the robot $(\mathbb{O}$. The maximum measurement range of MC3A is $100 \mathrm{lb}$; the sensitivity is 6 $\mu \mathrm{V} / \mathrm{V}-\mathrm{lb}$. The output voltage range of MC3A is of the range of microvolts, so an amplifier is required. The system uses a Texas Instruments INA128 instrumentation amplifier (D) for $20 \times$ amplification. The advantage of using the INA128 is that the minimum value of CMRR is 120 . It only requires a driving voltage of $2.25 \mathrm{~V}$, and it uses an Analog Devices OP177 OPA as an active low-pass filter to suppress noise $(\mathbb{E})$. The signal is amplified 50 times, and the cut-off frequency is $3 \mathrm{kHz}$. A National Instruments PCI-6034E data acquisition (DAQ) board is used to acquire a digital signal $(\mathbb{B}$. PCI-6034E is a 16-bit DAQ board, and the sampling rate is $200 \mathrm{kHz}$ ). The sampled data is displayed on a PC (G).

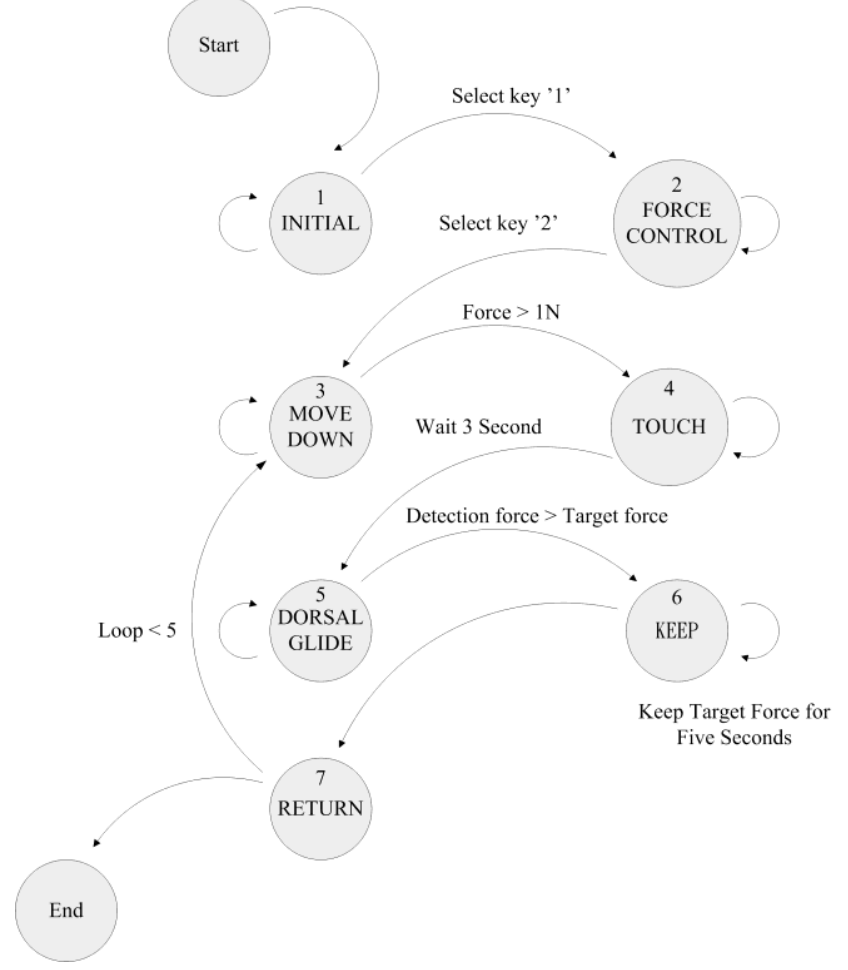

FIG. 4. The Algorithm State Machines Diagram.

The software control process of the PC program is shown in Fig. 4. There are seven program states in the complete process, and the functions of each state are as follows:

1) At the beginning, the program is in the INITIAL state. In this state, the parameters are initialized, such as the zero calibration of the sensing element and the setting of the target power, etc. After confirming the completion of data input, the user presses the " 1 " button and then leaves initial setting state.

2) In the force control state, the therapist can easily drive the robotic arm and guide it along a path. At this point, the path information will also be recorded to RAM and stored. After confirming the guiding path, the user presses the " 2 " button on the controller and the program enters the next state.

3) MOVE DOWN state. After the robotic arm reaches the position above the affected area, it will vertically move down along the world coordinate $\mathrm{Z}$ axis until the sensor shows that the change in force is more than $1 \mathrm{~N}$. The program will then judge the flange of the treatment touch for the affected areas. The program then enters State 4.

4) TOUCH state. When the robotic arm touches the affected areas, it will stop operation for three seconds, and proceed with the motions of State 5. This state aims to set a starting point on the data diagram and for the convenience to find the starting point of application of force to divide different data blocks and make data analyses easier.

5) DORSAL GLIDE state. The robotic arm will move down along the $\mathrm{Z}$ axis and maintain the same speed until the force detected by the sensor reaches the preset target force. The program then enters State 6.

6) KEEP state. When the force of the robotic arm reaches the target force, it needs to stabilize the same force for five seconds for joint laxity. The reaction force will decrease after continuously exerting pressure on human joints. During the five seconds, the program has to constantly adjust the position of the flange to maintain pressure within the range of the target force. After finishing, the program enters the next state.

The complete experimental framework platform is shown in Fig. 5. The rehabilitation robot can implement Grade I to Grade III joint mobilization and the PC will receive real-time data.

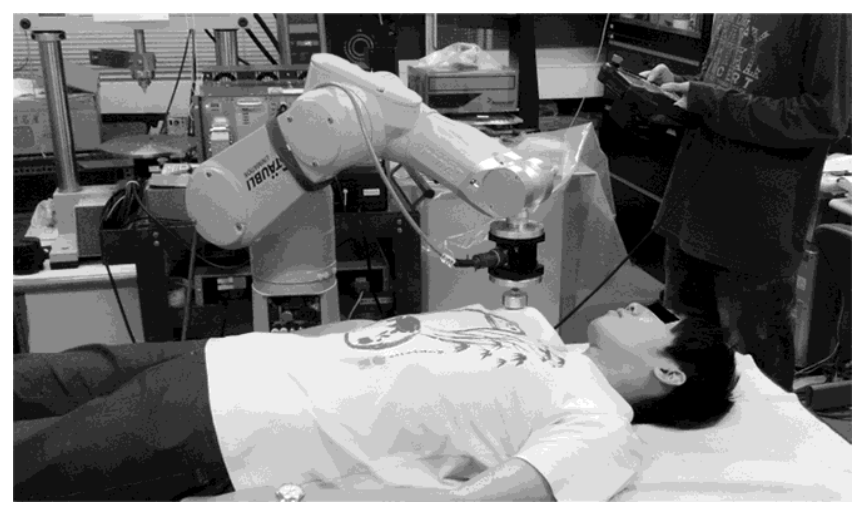

FIG. 5. Rehabilitation Robot Experiment Platform. 


\section{RESULTS AND DISCUSSION}

Fig. 6 shows how the GHJ rehabilitation robot conducts joint mobilization. The output force generated by the robot will displace the joints to reduce the resistance from the joint tissue of the frozen shoulder. The robot can set the strength of the force to 10-90 N. The robot motion speed is set at $1-10 \mathrm{~mm} / \mathrm{s}$, and the speed of data collection is $60-100 \mathrm{~Hz}$.

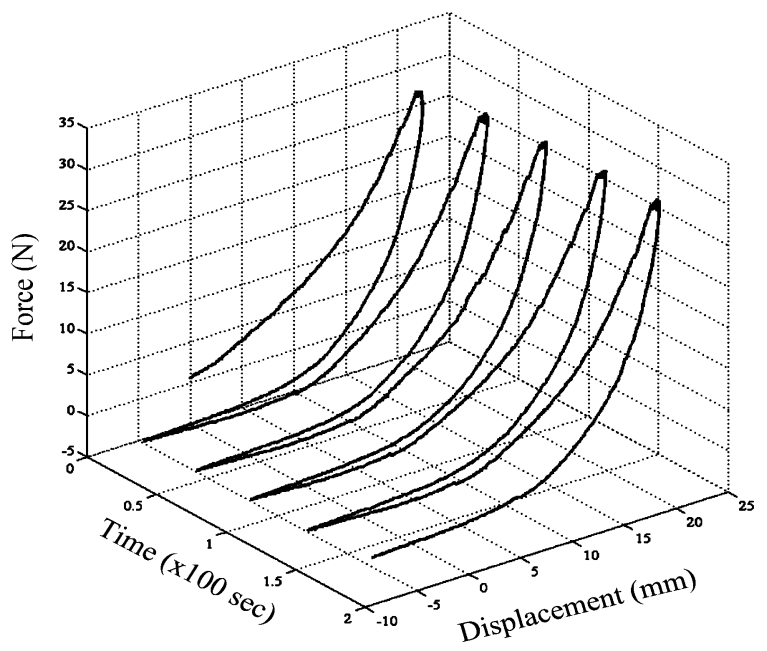

FIG. 6. The Display of Experiment Process of Rehabilitation Robot.

A load-displacement curve can be used to analyze the impedance of joint tissue. Fig. 7 shows that curve (1) will generate minimum displacement under the force of $30 \mathrm{~N}$, and the maximum displacement is $18.29 \mathrm{~mm}$. The rising speed is the fastest; that is, the impedance of the joint of Subject 1 is the maximum. Under the same force, curve (2) can generate more displacement; the maximum displacement of the joint of Subject 2 is $22.78 \mathrm{~mm}$. The displacement of curve (3) is the greatest, and the maximum displacement is $23.12 \mathrm{~mm}$; that is, the impedance of the joint of Subject 3 is the minimum. In curve 4 , the maximum displacement of Subject 4 is $21.03 \mathrm{~mm}$. In curve (5), at the beginning, the rising speed of the force is the slowest, so the position of IR is the farthest. Later, however, the rising speed is the fastest, so the distance of FR and IR is closer. The maximum displacement of the joint of Subject 5 is $21.90 \mathrm{~mm}$. The individual comparisons are shown in Fig. 8 , in which Subject 1, Subject 2, and Subject 4 progressed $0.67 \mathrm{~mm}, 0.77 \mathrm{~mm}$, and $0.59 \mathrm{~mm}$, respectively. Subject 3 progressed from $19.81 \mathrm{~mm}$ to $23.12 \mathrm{~mm}$, progressing the most with an increase of $3.31 \mathrm{~mm}$; this was followed by Subject 5, who progressed from $19.52 \mathrm{~mm}$ to $21.90 \mathrm{~mm}$, increasing by $2.38 \mathrm{~mm}$. Each subject exhibited significant improvement.

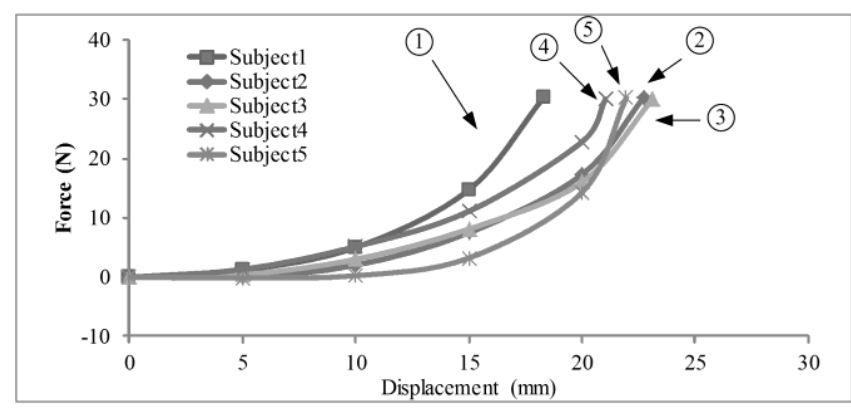

FIG. 7. Load-Displacement Curve.

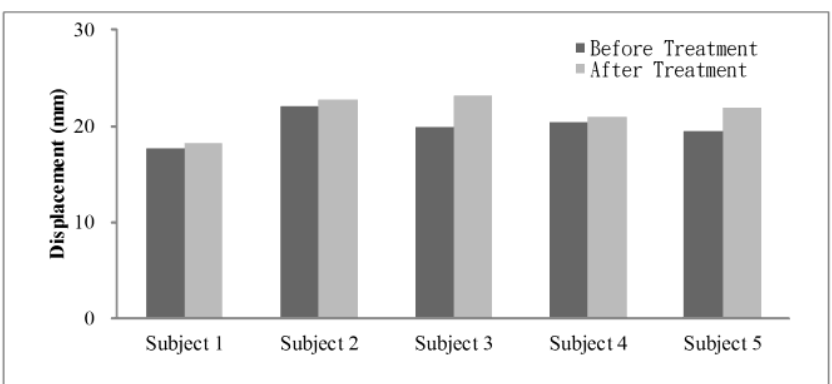

FIG. 8. Comparisons of Maximum Displacement Measured Before and After Treatment of Subjects.

\section{ACKNOWLEDGMENT}

This work was supported by the National Science Council, Taiwan, R.O.C. under contract No. NSC-102-2221-E-327030 .

\section{REFERENCES}

[1] H. Vermeulen, M. Stokdijk, P. Eilers, C. Meskers, P. Rozing, T. Vlieland, Ann. Rheum. Dis. 61, 115 (2002).

[2] C. C. Tai, J. R. Chang Chien, C. Y. Wang, L. C. Chen, C. H. Chou, and G. L. Wu, Biomed. Eng. Appl. Basis Comm. 20, 269 (2008).

[3] V. G. Popescu, G. C. Burdea, M. Bouzit, V. R. Hentz, IEEE Trans. Inf. Technol. Biomed. 4, 45 (2000).

[4] B. Y. Tseng, Principles and applications of digital signal controllers (ICCI Book, R.O.C, 2007).

[5] J. R. Chang Chien, G. H. Lin, A. T. Hsu, Rev. Sci. Instrum. 82, 105101 (2011). 